\title{
A new Parameterization for the Pion Vector Form Factor
}

\author{
C. Hanhart \\ Institut für Kernphysik, Institute for Advanced Simulation and Jülich Center for Hadron Physics, \\ Forschungszentrum Jülich, D-52425 Jülich, Germany
}

\begin{abstract}
A new approach to the parameterization of pion form factors is presented and for illustration applied to the pion vector form factor. It has the correct analytic structure, is at low energies consistent with recent high accuracy analyses of $\pi \pi$ scattering phase shifts and, at high energies, maps smoothly onto the well-known, successful isobar model.
\end{abstract}

Key words: Pion form factor, Omnès representation, 11.55.Bq, 13.40.Gp

\section{Introduction}

In recent years the knowledge about the low energy two-pion system has improved significantly, both experimentally as well as theoretically: for the low partial waves phase shift parameterizations of high accuracy exist from different dispersive analyses, either involving data only [1], or involving both data as well as constraints from chiral symmetry [2]. The analyses are based on Roy or Roy-type equations that respect analyticity as well as crossing symmetry. Especially, left-hand cuts are included without approximation.

In contrast to this, pion form factors or production reactions are often modeled either by sums of BreitWigners or improved versions thereof $[3,4]$ or by the $\mathrm{K}$-matrix formalism. In case of overlapping resonances unitarity gets violated by the former ansatz. The $\mathrm{K}$-matrix provides a clear improvement compared to the Breit-Wigner parameterization, since two-body unitarity is built in. However, in general analyticity is violated. On the one hand, in the standard treatment not the full dispersive corrections are considered (in the expressions for the self energies only the imaginary parts and their analytic continuation are being kept and not the full expressions - c.f. Eq. (11) below), although some works include them (see, e.g., Ref. [5]), on the other hand, the left hand cuts are not treated properly - if they are included at all, in order to fit the scattering amplitudes, they are often in the same way included in the production amplitude although there the left hand cuts are different or, as in case of form factors, even absent.

To be specific, in this work we focus on form factors and scattering with the goal to present simple formulas that allow for a data analysis that is consistent with analyticity and unitarity, however, without the necessity to solve dynamical equations. In addition, we present formulas that, by construction, in the low energy regime map smoothly and consistently onto what can be derived from the high accuracy analyses mentioned above and thus for the scattering even include the proper left hand cuts.

As an example and for demonstration we apply the formalism in this paper to the pion vector form factor, related to $\pi \pi$ scattering in the $p$-wave. The experimental situation for the $\rho$ resonances beyond the $\rho(770)$ is at present not very clear: different experiments find indications for different resonances - for a summary of the current situation see 'note on the $\rho(1450)$ and the $\rho(1700)^{\prime}$ ' in the Review of Particle Physics [6]. The formalism presented here could be an important step forward to clarify the situation for it allows for 


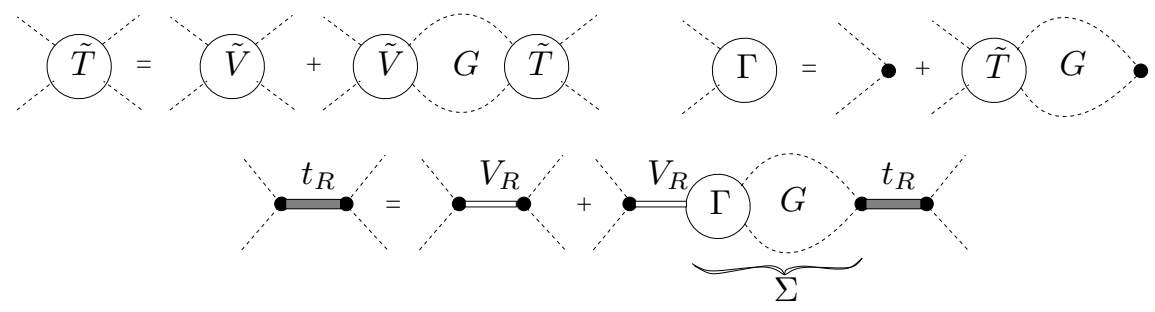

Fig. 1. Diagrammatic representation for the various ingredients of the formalism.

a simultaneous, consistent analysis of various channels/observables. To parameterize the vector form factor beyond $s=1 \mathrm{GeV}^{2}$ all studies agree on the need to include at least two resonances in addition to the $\rho(770)$, which is elastic. Thus, if we include one inelastic channel the pion vector form factor is parameterized in terms of in total 9 parameters -8 for the resonances and 1 additional parameter for the $\rho-\omega$ mixing (see Sec. 3). The number of parameters needed for each resonance agrees to standard parameterizations. The advantage of the parameterization presented here is that we do not need to approximate the left hand cuts, the consistency with low energy phase shift is ensured by construction and the connection between scattering and form factors is properly implemented. We found that once simultaneously to the form factor also the data on $e^{+} e^{-} \rightarrow$ non $2 \pi$ in the isovector state was fitted, the inclusion of not only an additional resonance but also of the $\pi \omega$ cahnnel together with a direct $\pi \pi \rightarrow \pi \omega$ coupling became necessary. This model, with in total 17 parameters, allowed for an acceptable fit to the data.

The paper is structured as follows: the most important formulas are motivated and presented in Sec. 2. Their more detailed derivation is given in Sec. 3. Results are presented in Sec. 4 and the paper closes with a short summary in Sec. 5. The two-potential formalism which forms the basis for the derivation is introduced in the Appendix.

\section{Summary of most important results}

When it comes to the interplay of resonance contributions and background terms the case of only one scattering channel is especially simple for here it is possible to give a closed form expression for the form factor $\mathcal{F}$ solely in terms of the elastic scattering phase shift - the so-called Omnès solution [7]. It is derived from a dispersion relation using the fact that

$$
\operatorname{disc}(\mathcal{F}(s))=2 i \sigma T(s)^{*} \mathcal{F}(s),
$$

where $T$ denotes the on-shell elastic scattering amplitude, and $\sigma=\sqrt{1-4 m^{2} / s}$ the two-body phase spacefor simplicity we assume the scattering particles to have equal mass $m$. Here 'disc' denotes the discontinuity of the form factor defined via

$$
\operatorname{disc}(\mathcal{F}(s))=\mathcal{F}(s+i \epsilon)-\mathcal{F}(s-i \epsilon)=2 i \operatorname{Im}(\mathcal{F}) .
$$

If the elastic phase shift is $\delta(s)$, with

$$
T(s)=\frac{1}{\sigma} \sin (\delta(s)) e^{i \delta(s)}
$$

then the form factor reads in the absence of bound states

$$
\mathcal{F}(s)=\xi(s) \Omega[\delta](s) P_{A}(s)
$$

with the Omnès function

$$
\Omega[\delta](s)=\exp \left\{\frac{s}{\pi} \int_{4 m^{2}}^{\infty} \frac{d s^{\prime}}{s^{\prime}} \frac{\delta\left(s^{\prime}\right)}{s^{\prime}-s}\right\},
$$

where the centrifugal barrier is introduced via the factor $\xi(s)={\sqrt{s-s_{\text {thr }}}}^{L}$ for $L$-waves, where $s_{\text {thr denotes }}$ the location of the threshold and the function $P_{A}(s)$ is a polynomial. Its degree may be fixed by the large $s$ behavior of the form factors. For values of $s<1 \mathrm{GeV}^{2}$ this methodology was used by various authors for the pion vector form factor, see, e.g. Refs. [4,8-13]. Here we will present a formalism that provides expressions 

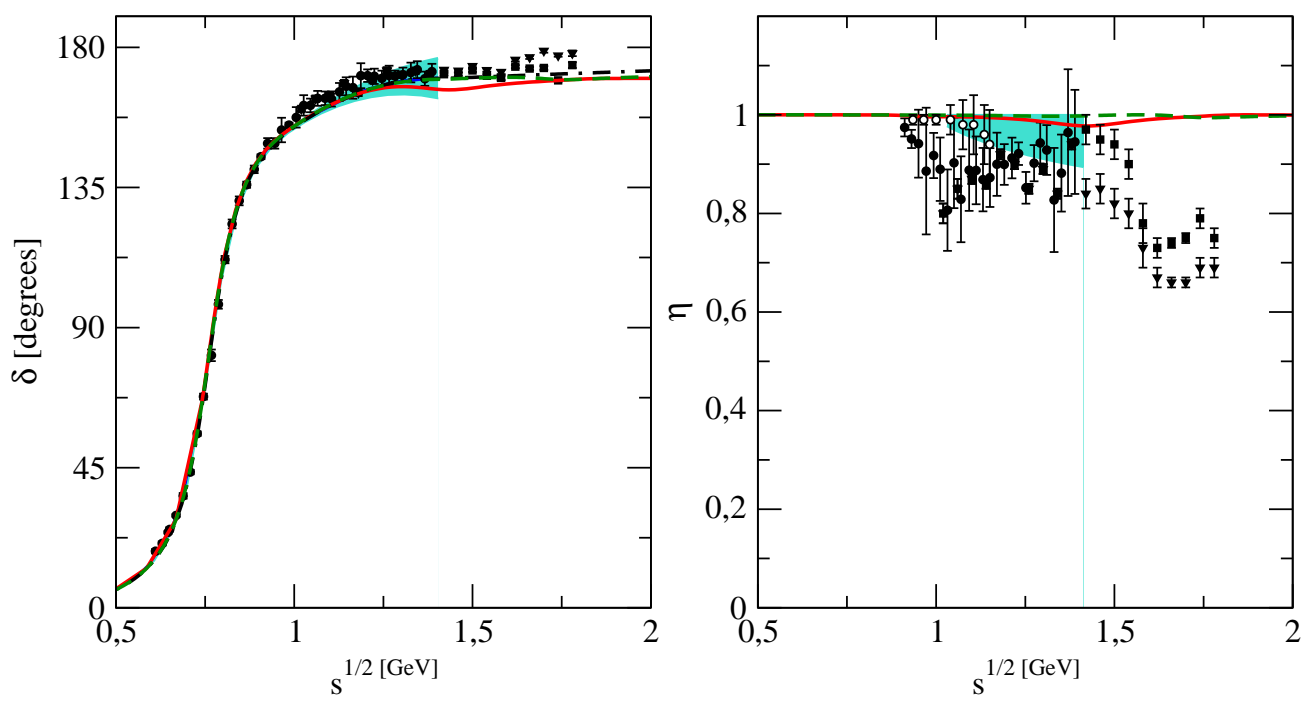

Fig. 2. Fits result for the pion $p$-wave phase shift (left panel) and inelasticity (right panel). The red solid (green dashed) line denotes the result of the fit \#2 (\#1). The dot-dashed line in the left panel refers to the input phase $\tilde{\delta}$. Data are from Ref. [14] (solid dots - only data below $1.4 \mathrm{GeV}$ are shown [15]), Ref. [16] (solid squares for solution (- - -); solid triangles for solution $(-+-))$, and Ref. [17] (open dots) and Ref. [1] (turquoise band).

that smoothly map onto Eq. (4) at lower energies while being an analytically improved version of the isobar model at higher energies.

Eqs. (1) and (4) apply only if the interactions are purely elastic - for the latter it is even necessary that they are elastic up to infinite energies. Clearly this is not realistic. However, experimental data show that at higher energies inelasticities are typically accompanied by resonances. We therefore split the full, partial wave projected, interaction potential $V$ into two pieces

$$
V(s)_{i j}=\tilde{V}(s)_{i j}+V_{R}(s)_{i j},
$$

where $i$ and $j$ denote the channels. The crucial feature for this approach is that the potential $\tilde{V}$ needs to be specified at no point. All what is needed are the corresponding phase shifts $\tilde{\delta}$. We now postulate the following properties:

- the potential $\tilde{V}$ is purely elastic, such that $\tilde{V}$ is non-vanishing only for $i=j=1$;

- deviations in the $\pi \pi$ phase-shifts from $\tilde{\delta}$ come either from $s$-channel resonances or via the coupling to inelastic channels;

- all long ranged forces are in the elastic $\pi \pi$ interactions $\tilde{V}$; all interactions in other channels are regarded as short ranged.

These are the model dependent assumptions of this approach (note, in case of the traditional isobar model one needs to assume that all interactions are mediated by $s$-channel resonances). Based on these Eqs. (1) and (4) can be easily generalized to multiple channels providing a convenient parameterization for both scattering as well as production amplitudes.

The full scattering $T$-matrix appears as the solution of a Bethe-Salpeter equation with input potential $V$ defined in Eq. (5). Using the two potential formalism (see Appendix) it is straightforward to derive the decomposition

$$
T(s)_{i j}=\delta_{i j} \delta_{1 i} \tilde{T}(s)+T_{R}(s)_{i j}=\delta_{i j} \delta_{1 i} \tilde{T}(s)+\Gamma_{\text {out }}(s)_{i} t_{R}(s)_{i j} \Gamma_{\text {in }}(s)_{j}^{\dagger}
$$

where $\tilde{T}$ is the purely elastic scattering $T$-matrix that derives from the potential $\tilde{V}$. For the vertex functions one has from time reversal invariance $\Gamma_{\text {out }}=\Gamma_{\text {in }}^{\dagger}$. They are, by assumption, non-trivial only in the $\pi \pi$ channel, where elastic scattering is mediated by $\tilde{T}$. As a result $\Gamma_{\text {out }}$ is diagonal. We may therefore write for any given partial wave 


$$
\tilde{T}=\frac{1}{\sigma_{1}} e^{i \tilde{\delta}} \sin (\tilde{\delta})
$$

and

$$
\Gamma_{\text {out }}(s)_{i}=\xi_{i}(s) \Omega[\tilde{\delta}](s) \text { for } \mathrm{i}=1(\pi \pi-\text { channel }) \text {; otherwise } \Gamma_{\text {out }}(s)_{i}=\xi_{i}(s),
$$

where $\xi_{i}(s){\sqrt{s-s_{\operatorname{thr} i}}}^{L}$ is the multichannel version of the centrifugal barrier factor given above. The resonance $T$ matrix $t_{R}$ may be written as

$$
t_{R}(s)_{i j}=\left[1-V_{R}(s) \Sigma(s)\right]_{i k}^{-1} V_{R}(s)_{k j}
$$

with the resonance potential (note: not all resonances are in $V_{R}$; elastic resonances may be included in $\tilde{T}$ as the $\rho(770)$ in the example below)

$$
\bar{V}_{R}(s)_{i j}=-\sum_{l=1}^{n} \frac{g_{i}^{(l)} g_{j}^{(l)}}{s-m_{(l)}^{2}} ; \quad V_{R}(s)=\bar{V}_{R}(s)-\bar{V}_{R}(0)+\gamma_{j} \delta_{i 1}+\gamma_{i} \delta_{j 1} .
$$

In Fig. 1 a graphic representation of the various quantities is given. The potential is subtracted at $s=0$ to ensure that the phase of the full $T$ matrix at low energies agrees to the input phase $\tilde{\delta}$. Clearly, the procedure does not guarantee a priori that the phase of the full $T$-matrix is close to that of $\tilde{T}$ in the whole range where $\tilde{T}$ is well determined, however, in practice this is indeed the case: as can be seen from the left panel of Fig. 2, at energies below $1 \mathrm{GeV}$ all curves shown, including that for the input phase shift, are indistinguishable.

The term containing the parameter $\gamma_{i}$, with $\gamma_{1}=0$, is included to allow for a direct transition of the $\pi \pi$ channel (channel number 1) to inelastic channels. For the pion vector form factor the fit to data requires such a coupling to the $\pi \omega$ channel - in effect it provides a direct $\rho \pi \omega$ coupling, which from phenomenology is known to be significant [18].

The self energy loop $\Sigma_{i}$ in channel $i$ appearing in Eq. (9) and Fig. 1 may be expressed via a twice subtracted dispersion relation (see Sec. 3 and Appendix) — one finds

$$
\Sigma_{i}(s)=\frac{s^{2}}{\pi} \int_{s_{\mathrm{thr}}}^{\infty} \frac{d s^{\prime}}{s^{\prime 2}} \frac{\sigma_{i}\left(s^{\prime}\right)\left|\Gamma_{i}\left(s^{\prime}\right)\right|^{2}}{s^{\prime}-s-i \epsilon} .
$$

For given interactions $T_{i j}$ it is straight forward to calculate the form factors $\mathcal{F}_{i}$. As shown in the next section, one finds

$$
\mathcal{F}(s)_{i}=\Gamma_{\text {out }}(s)_{i}\left[1-V_{R}(s) \Sigma(s)\right]_{i k}^{-1} M_{k},
$$

where the $M_{i}$ denote point like source terms for the production of particles into channel $i$. It may be written as

$$
M_{k}=c_{k}-\sum_{l=1}^{n} \frac{g_{i}^{(l)} \alpha^{(l)}}{s-m_{(l)}^{2}} .
$$

Since we assume that all interactions but those in the two-pion channel are driven by resonances, we choose $c_{k}=c \delta_{1 k}$, with some constant $c$. In case of the pion vector form factor, discussed in detail below, charge conservation demands $c=1$. In addition, to ensure the proper normalization of the form factor and to suppress the influence of the higher resonances on low energies, we use a photon-resonance coupling linear in $s^{1}$. In practice this means replacing $\alpha^{(l)}$ in Eq. (13) by $s \alpha^{(l)}$. The ingredients of the formalism are illustrated in Fig. 1.

All parameters introduced $-\kappa, g_{i}^{(l)}, \alpha^{(l)}, m_{(l)}, \gamma_{i}$ - are real as long as all (relevant) channels are treated explicitly as a consequence of time reversal invariance. Thus, in case of two channels, for each inelastic resonance we need to include 4 real parameters. Each additional channel adds in one more parameter per resonance. For the predominantly elastic resonances, which are included in the vertex functions $\Gamma$, the only free parameters are their couplings to the inelastic channels introduced via the $\gamma_{i}$.

Eq. (12) is the central result of our paper. If all vertex functions were chosen to be constant, it would reduce to the famous $P$-vector formalism [19]. However, since in our case $\Gamma_{1}$, which enters explicitly in the

\footnotetext{
1 On the Lagrangian level this means a coupling of the resonance to the photon field via $F^{\mu \nu} \partial_{\mu} V_{\nu}$ with $F^{\mu \nu}$ for the electromagnetic field strength tensor and $V_{\nu}$ for the resonance field.
} 

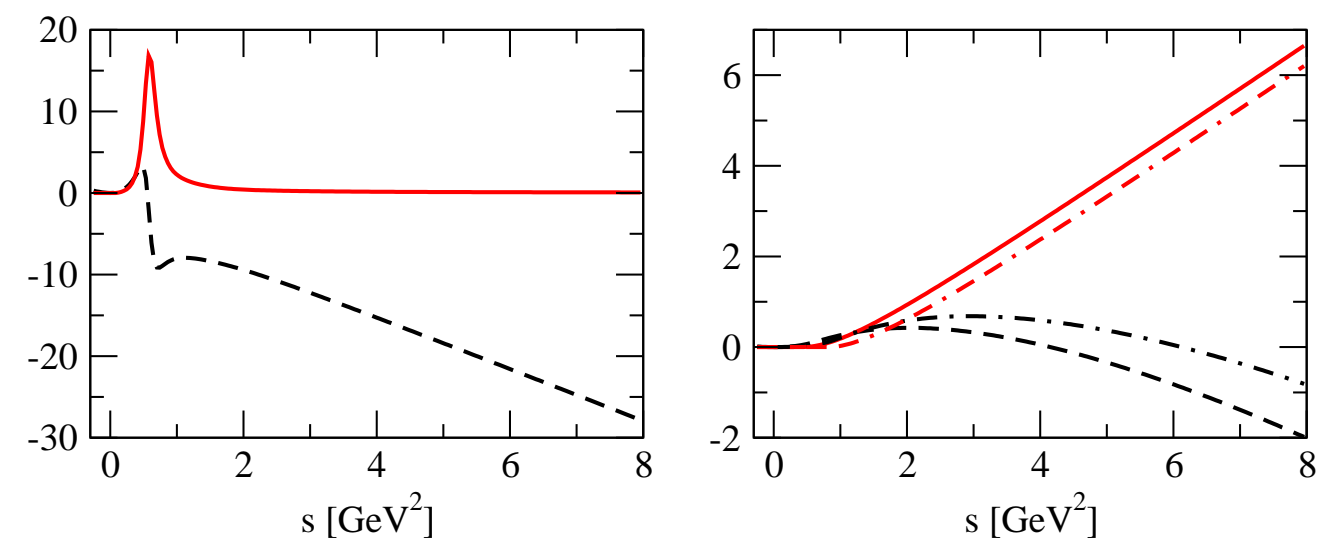

Fig. 3. Real and imaginary parts for the self energies $\Sigma_{i}(s)$ defined in Eq. (11). Left panel: self energy for the elastic channel, $\Sigma_{1}(s)$ - the solid (dashed) line shows the imaginary (real) part. Right panel: self energies for the inelastic channels. The solid (dashed) line shows the imaginary (real) part for the $4 \pi$ channel, while the dashed-dotted (double dashed-dotted) line shows the imaginary (real) part for the $\omega \pi$ channel.

expression for $\mathcal{F}_{1}$ as well as through $\Sigma_{1}$, is non-trivial, Eq. (12) provides a generalization to the conventional treatment.

\section{Derivation of the Formalism}

In this section the expressions presented in the previous section are derived. It is based on the twopotential formalism used, e.g., in Ref. [20] to control the Coulomb-nuclear interference and rederived in the notation used here in the Appendix. The important aspect to observe is that, although the derivation starts from a potential $\tilde{V}$, introduced in Eq. (5), the final expressions can all be expressed in terms of the corresponding scattering phase $\tilde{\delta}$.

The two-potential formalism splits the $T$-matrix into two pieces, the elastic $T$-matrix $\tilde{T}$ and the resonance $T$-matrix $T_{R}$ (c.f. Eq. (6)). The latter quantity is the solution of a Bethe-Salpeter equation derived from the resonance potential $V_{R}$, however, here the intermediate state free propagators, used in the standard treatment, are to be replaced by interacting propagators. This is achieved via inclusion of the vertex functions $\Gamma$ on the external legs as well as a proper modification of the intermediate meson loops connecting two insertions of the resonance potential - the so-called resonance self-energies $\Sigma_{i}$. It is straightforward to show that the imaginary parts of the self-energies read (see Appendix)

$$
\operatorname{Im}\left(\Sigma_{i}(s)\right)=\sigma_{i}\left|\Gamma_{i}(s)\right|^{2} .
$$

Thus the self energies can be calculated from a properly subtracted dispersion integral. Since $\Sigma\left(s_{0}\right)$ and $\partial \Sigma / \partial s\left(s_{0}\right)$ at some $s_{0}$ can be absorbed into the resonance masses and wave function renormalization constants (or, more appropriate for the present context: in the effective coupling constants of the resonances) we here use a twice subtracted version - c.f. Eq. (11). The self energy in the 2- $\pi$ channel needs as input only the vertex function $\Gamma_{1}(s)$ discussed in the previous paragraph. The resulting $\Sigma_{1}(s)$ is shown in the left panel Fig. 3.

To parameterize the inelastic channels we use for $i=2$ a structureless $4 \pi$ channel via the phase space factor $\sigma_{2}={\sqrt{1-16 m_{\pi}^{2} / s}}^{7}$, which provides the proper scaling of the four-body phase space near the threshold. For the barrier factor, appearing in Eqs. (8), we use $\xi_{2}=\sqrt{s-16 m_{\pi}^{2}}$. The resulting self energy $\Sigma_{2}(s)$ is shown in the right panel Fig. 3. Although the $\bar{K} K$ channel contributes significantly to isoscalar $\pi \pi$ interactions, it gives negligible contributions in the isovector channel [21]. Therefore in fit \#2 we include as additional inelastic channel $(i=3)$ the $\pi \omega$ channel. For a discussion on the possible role of the $\pi \omega$ channel on the $\pi \pi$ inelasticity see Ref. [22,23]. We take $\sigma_{3}=\lambda\left(s, m_{\omega}^{2}, m_{\pi}^{2}\right)^{1 / 2} / s$ and $\xi_{3}=\lambda\left(s, m_{\omega}^{2}, m_{\pi}^{2}\right)^{1 / 2}$. The resulting self energy $\Sigma_{3}(s)$ is also shown in the right panel Fig. 3. The linear rise of the imaginary part of both $\Sigma_{2}$ and $\Sigma_{3}$ comes from the centrifugal barrier terms $\xi_{2}$ and $\xi_{3}$, respectively. 
To calculate the form factor we may write (using the notation of the Appendix)

$$
\mathcal{F}=M+T G M,
$$

where $G_{i}$ is the operator representation for the integration over all intermediate $n$-body states of channel $i$ and the production vertices $M_{i}$ were defined in Eq. (13). Inserting Eq. (6) into Eq. (15), we get

$$
\mathcal{F}=M+\tilde{T} G M+T_{R} G M=\Gamma_{\text {out }}\left(1+t_{R} \Gamma_{\text {in }}^{\dagger} G\right) M .
$$

To proceed we may use the definition of the self energy, $\Sigma_{i}=\Gamma_{\text {in } i}^{\dagger} G_{i}$, to write

$$
t_{R} \Sigma=\left[1-V_{R} \Sigma\right]^{-1} V_{R} \Sigma=-1+\left[1-V_{R} \Sigma\right]^{-1} .
$$

Here we needed to assume that the range of interactions in the production vertex and in the vertex functions of the resonances is similar in all channels, for only then the same loop integral $\Sigma_{i}$ can be used as self energy contribution for the resonances as well as convolution integral of $M_{i}$ and the resonance potential. From this we get

which agrees to Eq. (12).

$$
\mathcal{F}=\Gamma_{\text {out }}\left[1-V_{R} \Sigma\right]^{-1} M,
$$

It is important to observe that the expression given in Eq. (12) is consistent with the coupled channel version of the unitarity relation for form factors, Eq. (1), since

$$
\begin{aligned}
\operatorname{disc}(\mathcal{F}) & =\operatorname{disc}\left(\Gamma_{\text {out }}\right)\left[1-V_{R} \Sigma\right]^{-1} M+\Gamma_{\text {out }}^{*} \operatorname{disc}\left(\left[1-V_{R} \Sigma\right]^{-1}\right) M \\
& =2 i \tilde{T}^{*} \sigma \Gamma_{\text {out }}\left[1-V_{R} \Sigma\right]^{-1} M+\Gamma_{\text {out }}^{*}\left[1-V_{R} \Sigma^{*}\right]^{-1} V_{R} \operatorname{disc}(\Sigma)\left[1-V_{R} \Sigma\right]^{-1} M \\
& =2 i \underbrace{\left(\tilde{T}^{*}+\Gamma_{\text {out }}^{*}\left[1-V_{R} \Sigma^{*}\right]^{-1} V_{R} \Gamma_{\text {out }}^{*}\right)}_{T^{*}} \sigma \underbrace{\Gamma_{\text {out }}\left[1-V_{R} \Sigma\right]^{-1} M}_{\mathcal{F}}
\end{aligned}
$$

where in the intermediate step the unitarity relation for the vertex function, Eq. (1), and the self energy, Eq. (14), were used.

An interesting observable is the ratio $r$ of the total cross section for $e^{+} e^{-}$annihilation into hadronic states with $I=1$ other than $\pi^{+} \pi^{-}$over $\sigma_{e^{+} e^{-} \rightarrow \pi^{+} \pi^{-}}$- a compilation of this quantity can be found in Ref. [21]. In this ratio the unitarization effects in the resonance $T$-matrix cancel largely. For example, in case of only one inelastic channel we get

$$
r=\left|\left(\frac{\sigma_{2} \Gamma_{\text {out } 2}}{\sigma_{1} \Gamma_{\text {out } 1}}\right) \frac{\left(1-V_{R 11} \Sigma_{1}\right) M_{2}+V_{R 12} \Sigma_{1} M_{1}}{\left(1-V_{R 22} \Sigma_{2}\right) M_{1}+V_{R 12} \Sigma_{2} M_{2}}\right|^{2},
$$

clearly being very directly sensitive to the resonance parameters.

The vector form factor in the two pion channel is directly accessible from two reactions, namely from $e^{+} e$-annihilation and from $\tau$ decays. In the former case, in addition to what was discussed so far, the isospin violating mechanism of $\rho-\omega$ mixing needs to be included. It is visible as a striking narrow structure close to the peak of the corresponding form factor (c.f. inlay in Fig. 4). The inclusion of this mixing in the present formalism is straight forward - we here use a slightly modified version to what is used in Ref. [11], namely, for the neutral, $\pi^{+} \pi^{-}$, channel

$$
F_{1}(s) \longrightarrow F_{1}(s)\left(1+\kappa \frac{s}{s-m_{\omega}^{2}+i m_{\omega} \Gamma_{\omega}}\right) .
$$

We here use $m_{\omega}=0.7826 \mathrm{GeV}$ and $\Gamma_{\omega}=0.0085 \mathrm{GeV}$. The strength parameter $\kappa$ is part of the fit. The only difference between the form factors in the neutral (from $e^{+} e^{-}$annihilation) and in the charged (from $\tau$ decays) channel is that in the latter we choose $\kappa=0$.

\section{Results for the pion phases, inelasticities and form factors in the $p$-wave}

To be specific we now focus on the pion vector form factor. The resonance parameters will be determined by a fit to data on the pion vector form factor as well as inclusive data on inelastic channels. In addition we need as input the elastic phase shifts $\tilde{\delta}$, which largely fix the properties of the $\rho(770)$. 

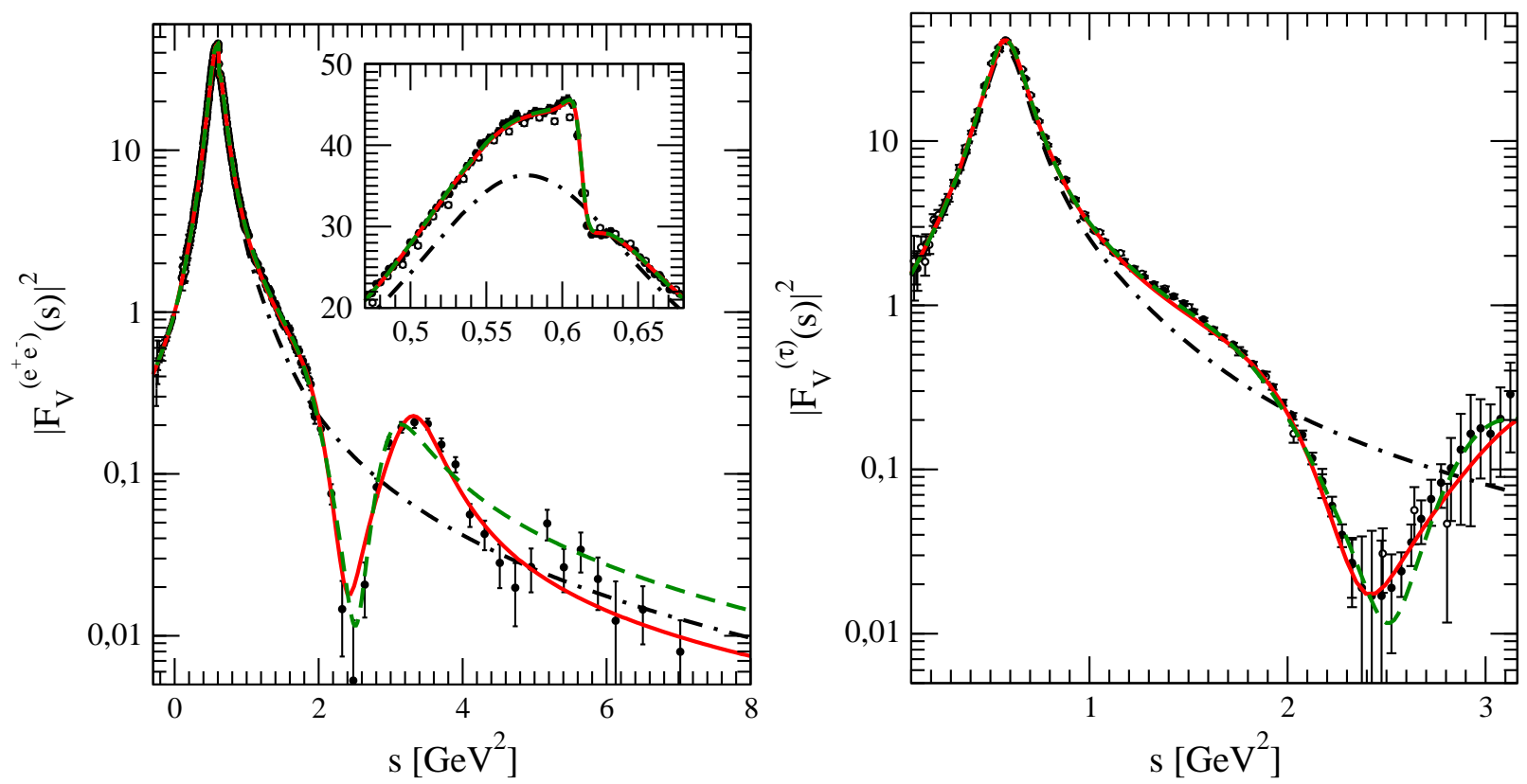

Fig. 4. Fit result for the pion vector form factor $F_{V}=\mathcal{F}_{1} / \xi_{1}$. Left panel: For the neutral channel. The red solid (green dashed) line denotes the result of fit \#1 (\#2). The black dot-dashed line shows the form factor derived from the Omnès function only. Data are from the reaction $e^{+} e^{-} \rightarrow \pi^{+} \pi^{-}$presented in Refs. [24-26]. Right panel: For the charged channel. Data are from Belle [27] and CLEO [28].

\begin{tabular}{|c|c|c|c|c|c|c|c|c|c|c|c|c|c|c|c|c|c|}
\hline fit & $\kappa \times 10^{3}$ & $m_{(1)}$ & $m_{(2)}$ & $m_{(3)}$ & $\gamma_{3}$ & $g_{1}^{(1)}$ & $g_{2}^{(1)}$ & $g_{3}^{(1)}$ & $g_{1}^{(2)}$ & $g_{2}^{(2)}$ & $g_{3}^{(2)}$ & $g_{1}^{(3)}$ & $g_{2}^{(3)}$ & $g_{3}^{(3)}$ & $\alpha^{(1)}$ & $\alpha^{(2)}$ & $\alpha^{(3)}$ \\
\hline$\# 1$ & $-1.95(4)$ & $1.5(1)$ & $2.2(1)$ & - & 0 & $0.020(2)$ & $0.65(1)$ & - & $0.25(2)$ & $-1.8(2)$ & - & - & - & - & $3.1(2)$ & $1.4(1)$ & - \\
$\# 2$ & $-1.93(3)$ & $1.2(1)$ & $1.6(1)$ & $4.7(4)$ & $-0.06(1)$ & $0.0(7)$ & $1.3(2)$ & $0.5(1)$ & $0.10(2)$ & $0.0(4)$ & $-0.8(2)$ & $0.5(1)$ & $-1(1)$ & $-12(2)$ & $0.0(1)$ & $-0.4(2)$ & $5.2(2)$ \\
\hline
\end{tabular}

\section{Table 1}

Parameter values for the mixing parameter $\kappa$ and the resonance parameters for the two fits performed. The uncertainties listed refer to the statistical uncertainty of the fit only. Masses and $\gamma_{3}$ are given in $\mathrm{GeV}$ and $\mathrm{GeV}^{-2}$, respectively; all other couplings are dimensionless.

For our work we will use for energies below $s_{\text {cut }}=1.4^{2} \mathrm{GeV}^{2}$ the central values for the phase shift provided in Ref. [1] — see Eqs. (A7) and (A8) therein. For energies above this value we smoothly extrapolate the phase shift to a value of $\pi$ via

$$
\tilde{\delta}(s)=\pi+\left(\tilde{\delta}\left(s_{\text {cut }}\right)-\pi\right)\left(\frac{\Lambda^{2}-s_{\text {cut }}}{\Lambda^{2}-s}\right) .
$$

It turns out that for $\Lambda \geq 2 \mathrm{GeV}$ the results are basically insensitive to the actual value used ${ }^{2}$. We thus chose $\Lambda=10 \mathrm{GeV}$ in what follows. The asymptotic value $\pi$ for the phase ensures that the vertex function $\Gamma_{1}(s)$ decreases as $1 / s$ as demanded for the vector form factor. The resulting elastic $\pi \pi$ phase shifts shown as the black dot-dashed line in the left panel of Fig. 2. The form factor from the Omnés function alone is shown as the black dot-dashed line in Figs. 4. It provides an acceptable description of the data up to $s=1$ $\mathrm{GeV}^{2}$, although there are some deviations visible (see inlay in the left panel). At higher energies a significant deviation becomes visible.

Two different fits were performed using the MINUIT package of the CERN library: the goal of fit \#1 is to find the minimal parameter set necessary to get an acceptable description of the pion vector form factor. In line with previous studies we find that at least two resonances and one inelastic channel are needed. In addition we may chose all $\gamma_{i}=0$ (c.f. Eq. (10)). The fit result is shown as the dashed green line in Fig. 4

$\overline{2}$ This is correct up to an unphysical pole located at $s=-\Lambda^{2}$, which, however, does not influence visibly the amplitude for $s>-\Lambda^{2}$. 


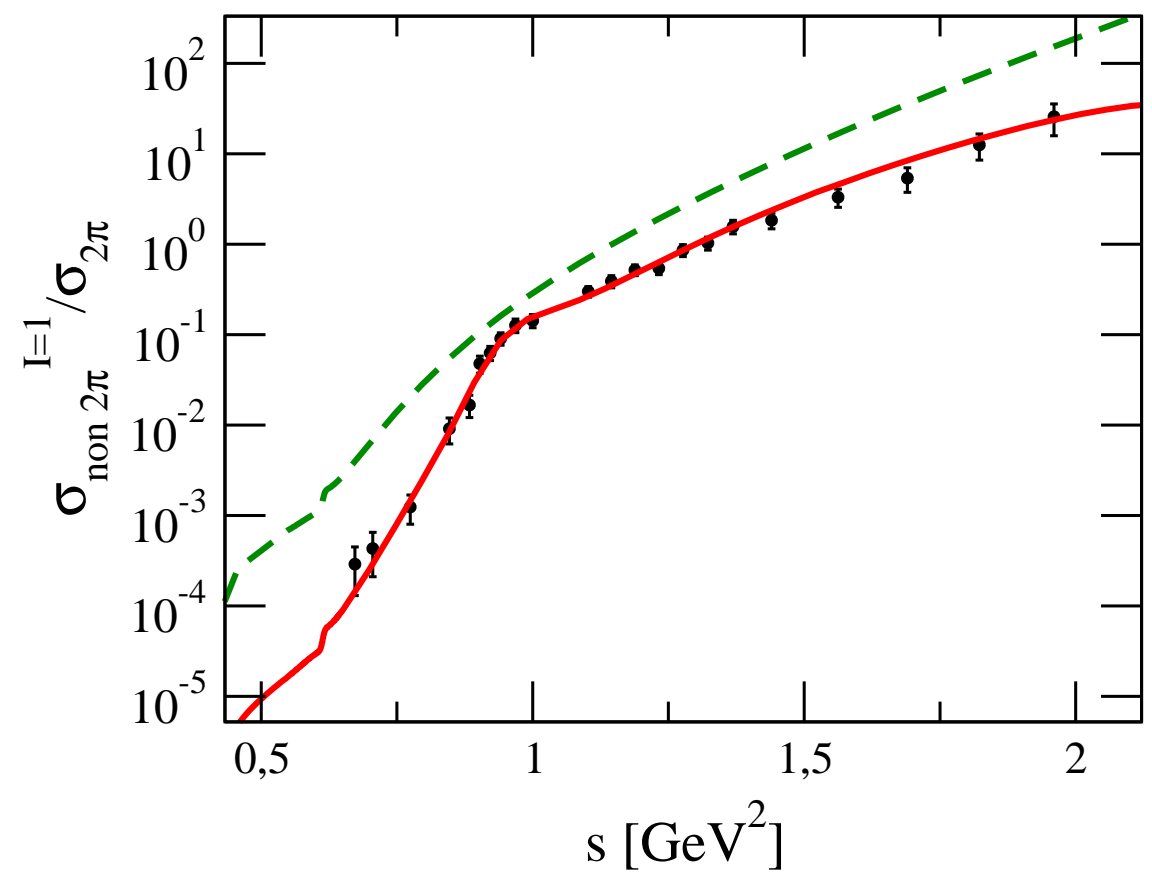

Fig. 5. Results for the ratio $r=\sigma_{e^{+} e^{-} \rightarrow(\operatorname{non} 2 \pi)}^{\mathrm{I}} / \sigma_{e^{+} e^{-} \rightarrow \pi^{+} \pi^{-}}$. The red solid (green dashed) line denotes the result of the fit \#2, (\#1). Data are from the compilation of Ref. [21]. The small step at $s=0.6 \mathrm{GeV}^{2}$ results from $\rho$ - $\omega$ mixing present in the denominator of $r$.

for the pion vector form factor and in Fig. 2 for $p$-wave phases and inelasticity. However, it turned out that with this parameter set it was impossible to simultaneously also describe $r$ - see green dashed line in Fig. 5 . Therefore we performed a second fit, fit \#2, with the goal to get a decent description of both $F_{V}$ as well as $r$. This called for an inclusion of 2 inelastic channels - in addition to the $4 \pi$ channel already included in fit \#1 we now also include the $\pi \omega$ channel -3 resonances as well as a non-vanishing value of $\gamma_{3}$. Fit \#2 has 17 adjustable parameters. The fit results are shown as the red solid lines in Figs. 2, 4 and 5 for phases and inelasticities, the pion vector form factor from $e^{+} e^{-}$annihilation and $\tau$ decays, and the ratio $r$, respectively. Note, in case of the pion vector form factor we fit to the BaBar data on $e^{+} e^{-}$annihilation only, for it extends to higher energies ${ }^{3}$. Especially, the $\tau$ data are not included in the fit - the result comes out as a prediction. The parameters determined in both fits are given in Tab. 1. It is important to note that for the pion vector form factor alone both fits are of similar quality: for fit \#1 and \#2 we have $\chi^{2} /$ d.o. $f=1.3$ and 1.2 , respectively. However, only fit \#2 provides an acceptable description for $r$. This result nicely illustrates that one should not analyze the pion vector form factor without looking at the non $2 \pi$ channels at the same time - to our knowledge in this work a combined analysis was performed for the first time. Note that at $s=2 \mathrm{GeV}^{2}$ Ref. [21] reports a value of $r \sim 26$, which shows that already at this relatively low energy the $2 \pi$ channel provides only a small fraction of the $e^{+} e^{-}$annihilation rate in the $I=1$ channel.

The $s$ dependence of the non $2 \pi$ data shown in Fig. 5 calls at least for two inelastic channels, since there is a change in slope visible at around $s \sim 0.9$. In fit \#2 this is accounted for by the inclusion of the $\pi \omega$ channel. However, even this three channel fit is still too simplified, for there should be not only correlations amongst the 4 pions in channel 2 included, e.g. from $a_{1} \pi, \rho \rho$, and $\rho \sigma$. In addition there are also channels like $\eta \pi \pi$. The data included in the current study does not allow one to disentangle these and therefore to improve the description of $r$ further. What is necessary is an inclusion of the large number of exclusive measurements available from $e^{+} e^{-}$annihilation. We leave this to a future study.

3 In this exploratory study we regard this as appropriate since in this work we do not perform an uncertainty estimate of the parameters extracted. 

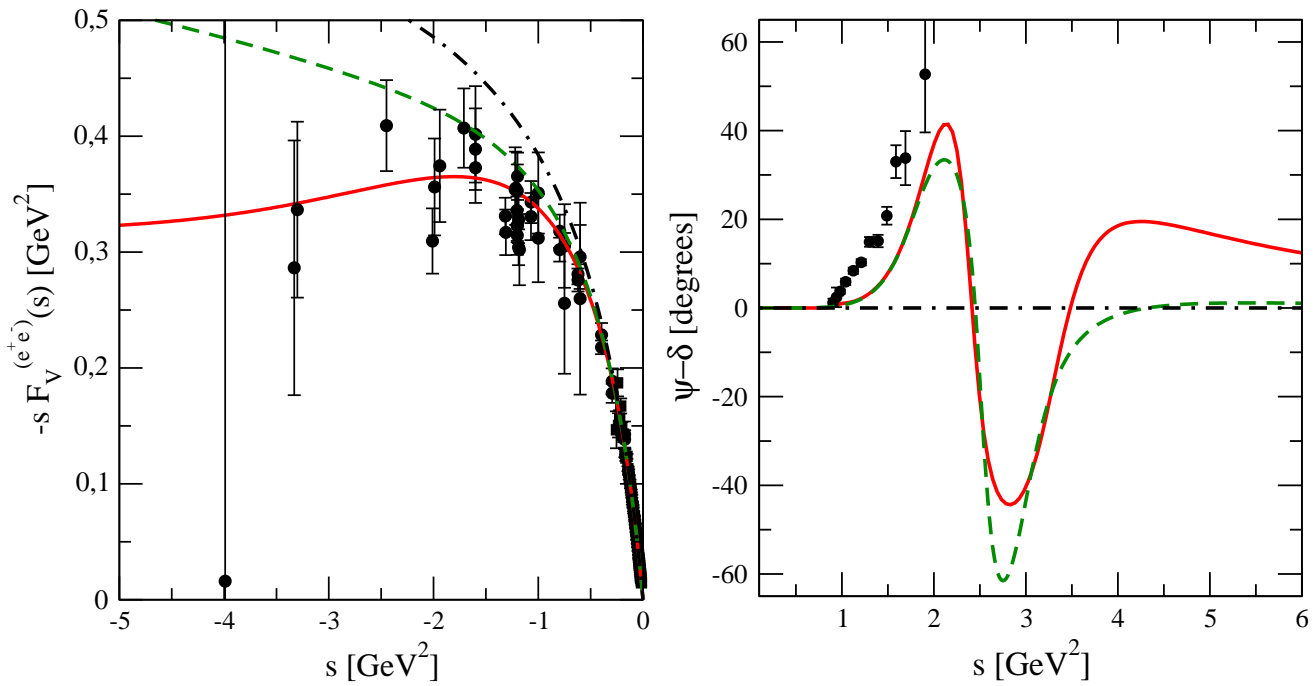

Fig. 6. Left panel: Fit result for the pion vector form factor at space-like energies. The lines are the same as in Fig. 4. Data are from Ref. [29,26]. Right panel: phase difference between $\psi$, the phase of the form factor, and the scattering phase shift $\delta$. The data shown indicate the upper bound of the phase shift difference presented in Ref. [21].

It is important to stress that the mass parameters given in the table are bare parameters that get renormalized by the self energies. It is therefore possible that the resonance poles come out similar in both fits, for the unitarization effects are different. However, we postpone the determination of pole positions and residues, which requires the evaluation of the elastic $T$-matrix $\tilde{T}$ in the complex plane, to a later work.

At small, space like energies the results of the two fits give identical results, c.f. left panel of Fig. 6 . Both lead to a pion radius slightly enhanced compared to what comes from the Omnès function itself, shown by the dot-dashed line: through the inclusion of the high-lying resonances the mean square charge radius of the pion increases by nearly $10 \%$ from $0.40 \mathrm{fm}^{2}$ to $0.44 \mathrm{fm}^{2}$. The latter value is consistent with the values extracted in Refs. [30,31]. For the curvature we find $c_{V}=3.9 \mathrm{GeV}^{-4}$, in line with Refs. [13,32]. The effect of the higher resonances is quantitatively in line with expectations from dimensional analysis that predicts an effect on the mean square radius of order of the square of the inverse resonance mass $\sim 0.02 \mathrm{fm}^{2}$. At higher space like energies the results move appart from each other. While fit \# 2 is consistent with the largely model independent bounds for the form factor derived in Ref. [33], fit \# 1 (the form factor from the Omnes function) is at most marginally consistent (inconsistent).

The left panel of Fig. 2 nicely illustrates that within the formalism presented the high accuracy phase shifts up to $1 \mathrm{GeV}$ are reproduced very well. One also finds that the phase shifts for the full model largely agree to the input phase in the whole energy range considered (this is not the case for the phase of the form factor, as discussed in the next paragraph) as well as to the data of Ref. $[14,16]^{4}$. This happens, since the resonance couplings to the $\pi \pi$ channel are rather small - the resonances show up prominently in the form factor only due to large couplings to the photon. Especially, the present model can not account for the significant inelasticity visible in the data of Ref. [16] — the according to Ref. [34] preferred solution $(-+-)$ shows an inelasticity of the 0.8 already at $1 \mathrm{GeV}$. At this point in time it is not possible to decide whether this failure is an indication of a short coming of the model used here, or of the data of Refs. [14,16]. What might support the latter conjecture is that the data on $\eta$ of Refs. $[14,16]$ are in disagreement with both the analysis of Ref. [17] as well as that of Ref. [1] at around $1 \mathrm{GeV}$.

In the elastic regime the phase of the form factor has to agree to the phase of elastic scattering - a fact known as Watson theorem. At higher energies this connection is lost. In the right panel of Fig. 6 we show the difference between $\psi$, the phase of the form factor, and the scattering phase shift $\delta$. Also shown in the panel is the allowed upper bound of the phase shift difference given in Ref. [21]. As one can see our amplitudes

\footnotetext{
4 Only two of the 4 solutions presented in that paper are given, for the other two are in strong discrepancy with the phase shifts of Ref. [1].
} 
largely exhaust the range allowed by unitarity. This reflects again the fact that in the present formalism all resonances besides the $\rho(770)$ couple to elastic scattering only weakly.

\section{Summary and Outlook}

In this paper a formalism was presented that allows for a simultaneous description of both $\pi \pi$ scattering data as well as form factors without the need to model the low energy regime: at low energies $\pi \pi$ phases can be used as input directly. At higher energies the formalism maps smoothly onto the well known $N / D$ method which is similar to the $K$-matrix approach, however, with improved analytical properties. As an example in this paper the formalism was applied to pion pairs in the $p$-wave. An excellent description is found for the pion vector form factor in both the neutral channel - from $e^{+} e^{-}$annihilations, with $\rho-\omega$ mixing included - as well as the charged channel - from $\tau$ decays. In addition we also found a qualitative agreement with data on the non $2 \pi$ channels from $e^{+} e^{-}$annihilations - these data were studied within a dynamical model here for the first time.

We found, however, that within the given formalism it was not possible to to describe the behavior of the inelasticity given in Refs. $[14,16]$. At this point in time we are not able to judge if this deviation indicates a short coming of the model or points at a problem in the data. However, the observation that the values of $\eta$ of Refs. [14,16] are in disagreement with the analyses of Refs. [1,17] at $s \sim 1 \mathrm{GeV}^{2}$ might indicate that there is a problem in the data of Refs. $[14,16]$ also at higher energies.

The formalism described here can be applied to all partial waves, especially also the isoscalar $s$-wave. Here, however, it is less clear what to use for the elastic phase shift $\tilde{\delta}$, since the pronounced structure from the $f_{0}(980)$, which also couples strongly to $\bar{K} K$, shows up already short after the phase reached $90^{\circ}$. We leave this study to a future work.

\section{Acknowledgment}

I thank Simon Eidelman, Martin Hoferichter, Bastian Kubis, Ulf-G. Meißner and Juan M. Nieves for useful and inspiring discussions and comments to the manuscript and Wolfgang Ochs for useful remarks about the data of Refs. $[14,16]$.

\section{Appendix A. The two potential formalism}

Let us assume that there is a sensible way to split the scattering potential into two pieces (as in the main text the potentials $\tilde{V}$ and $V_{R}$, the vertex function $\Gamma$ as well as the T-matrices are matrices in channel space, while the form factor $F$ and the production vertices $M$ are vectors in channel space)

$$
V=\tilde{V}+V_{R} .
$$

We will show in this Appendix that the full $T$-matrix can be split accordingly. In operator form the BetheSalpeter equation for the $T$ matrix may be written as

$$
T=V+V G T=\tilde{V}+V_{R}+\left(\tilde{V}+V_{R}\right) G T .
$$

Here $G_{i}$ denotes the operator for the integral over the $n$-particle intermediate state of channel $i$, e.g. for the two- $\pi$ intermediate state we have

$$
V G V \propto \frac{1}{i} \int \frac{d^{4} k}{(2 \pi)^{4}} V(k, . .) \frac{1}{k^{2}-m^{2}+i \epsilon} \frac{1}{(k-P)^{2}-m^{2}+i \epsilon} V(k, . .),
$$

where $P$ denotes the total $4-$ momentum of the system, $P^{2}=s$. Note, not all arguments of the potential $V$ are shown explicitly. Introducing $\tilde{T}$ as the solution of

$$
\tilde{T}=\tilde{V}+\tilde{V} G \tilde{T}
$$

and the dressed vertex functions (to simplify notations, in the appendix we do not show the centrifugal barrier factors $\xi$ explicitly)

$$
\Gamma_{\text {out }}=1+\tilde{T} G \text { and } \Gamma_{\text {in }}^{\dagger}=1+G \tilde{T}
$$


we get

$$
T=\tilde{T}+T_{R}=\tilde{T}+V_{R} \Gamma_{\text {in }}^{\dagger}+\left(V_{R} G+\tilde{V} G\right) T_{R} .
$$

Due to time reversal invariance we have $\Gamma_{\text {out }}=\Gamma_{\text {in }}^{\dagger}$. Since $\operatorname{disc}(G)=2 i \sigma$ and $\operatorname{disc}(\tilde{T})=2 i \sigma \tilde{T}^{*} \tilde{T}$ one has

$$
\operatorname{disc}\left(\Gamma_{\text {out }}(s)\right)=\operatorname{disc}(\tilde{T}) G+\tilde{T}^{*} \operatorname{disc}(G)=\sigma \tilde{T}(s)^{*} \Gamma_{\text {out }}(s)
$$

thus $\Gamma_{\text {out }}$ holds the unitarity relation for a form factor in a channel where the interactions are given by $\tilde{T}$.

We may therefore define

$$
T_{R}=\Gamma_{\text {out }} t_{R} \Gamma_{\text {in }}^{\dagger}
$$

and derive with $\Sigma=G \Gamma_{\text {out }}$ and $\tilde{V} G \Gamma_{\text {out }}=\Gamma_{\text {out }}-1$

$$
t_{R}=V_{R}+V_{R} \Sigma t_{R} \longrightarrow t_{R}=\left[1-V_{R} \Sigma\right]^{-1} V_{R} .
$$

From the definition above the discontinuity of the self-energy $\Sigma$ is found to be

$$
\operatorname{disc}(\Sigma)=\operatorname{disc}(G) \Gamma_{\text {out }}+G^{*} \operatorname{disc}\left(\Gamma_{\text {out }}\right)=2 i \underbrace{\left(1+G^{*} \tilde{T}^{*}\right)}_{\Gamma_{\text {out }}^{*}} \sigma \Gamma_{\text {out }},
$$

which was used to derive Eq. (11).

\section{References}

[1] R. Garcia-Martin et al., Phys. Rev. D 83 (2011) 074004 [arXiv:1102.2183 [hep-ph]].

[2] G. Colangelo, J. Gasser and H. Leutwyler, Nucl. Phys. B 603 (2001) 125 [hep-ph/0103088].

[3] G. J. Gounaris and J. J. Sakurai, Phys. Rev. Lett. 21 (1968) 244.

[4] M. F. Heyn and C. B. Lang, Z. Phys. C 7 (1981) 169.

[5] A. V. Anisovich et al., Phys. Rev. D 84 (2011) 076001.

[6] K. Nakamura et al. [Particle Data Group Collaboration], J. Phys. G G 37 (2010) 075021.

[7] R. Omnes, Nuovo Cim. 8 (1958) 316.

[8] J. Gasser and U.-G. Meißner, Nucl. Phys. B 357 (1991) 90.

[9] F. Guerrero, Phys. Rev. D 57 (1998) 4136 [arXiv:hep-ph/9801305]; F. Guerrero and A. Pich, Phys. Lett. B 412 (1997) 382 [arXiv:hep-ph/9707347].

[10] A. Pich and J. Portoles, Phys. Rev. D 63 (2001) 093005 [hep-ph/0101194].

[11] J. F. De Troconiz and F. J. Yndurain, Phys. Rev. D 65 (2002) 093001 [hep-ph/0106025].

[12] F. Guerrero and J. A. Oller, Nucl. Phys. B 537 (1999) 459 [Erratum-ibid. B 602 (2001) 641] [hep-ph/9805334].

[13] F. -K. Guo et al., Phys. Lett. B 678, 90 (2009) [arXiv:0812.3270 [hep-ph]].

[14] B. Hyams et al., Nucl. Phys. B 64 (1973) 134.

[15] W. Ochs, private communication. See also Ref. [16].

[16] B. Hyams et al., Nucl. Phys. B 100 (1975) 205.

[17] S. D. Protopopescu et al., Phys. Rev. D 7 (1973) 1279.

[18] M. Gell-Mann, D. Sharp and W. G. Wagner, Phys. Rev. Lett. 8 (1962) 261.

[19] I. J. R. Aitchison, Nucl. Phys. A 189 (1972) 417.

[20] K. Nakano, Phys. Rev. C 26 (1982) 1123.

[21] S. Eidelman and L. Lukaszuk, Phys. Lett. B 582, 27 (2004) [hep-ph/0311366].

[22] B. Costa de Beauregard, et al., Phys. Lett. B 67 (1977) 213.

[23] F. Niecknig, B. Kubis and S. P. Schneider, arXiv:1203.2501 [hep-ph].

[24] B. Aubert et al. [BABAR Collaboration], Phys. Rev. Lett. 103 (2009) 231801 [arXiv:0908.3589 [hep-ex]].

[25] F. Ambrosio et al. [KLOE Collaboration], Phys. Lett. B 700 (2011) 102 [arXiv:1006.5313 [hep-ex]].

[26] S.R. Amendolia et al. [NA7 Collaboration], Nucl. Phys. B 277 (1986) 168.

[27] M. Fujikawa et al. [Belle Collaboration], Phys. Rev. D 78 (2008) 072006 [arXiv:0805.3773 [hep-ex]].

[28] S. Anderson et al. [CLEO Collaboration], Phys. Rev. D 61 (2000) 112002 [hep-ex/9910046].

[29] G. M. Huber et al. Phys. Rev. C 78 (2008) 045203; H. Ackermann et al., Nucl. Phys. B 137 (1978) 294. J. Volmer et al. Phys. Rev. Lett. 86 (2001) 1713; C. J. Bebek et al., Phys. Rev. D 17 (1978) 1693.

[30] F. Stollenwerk et al., Phys. Lett. B 707 (2012) 184 [arXiv:1108.2419 [nucl-th]].

[31] G. Colangelo, Nucl. Phys. Proc. Suppl. 131 (2004) 185 [hep-ph/0312017].

[32] G. Abbas, B. Ananthanarayan and S. Ramanan, Eur. Phys. J. A 41 (2009) 93 [arXiv:0903.4297 [hep-ph]].

[33] B. Ananthanarayan, I. Caprini and I. S. Imsong, arXiv:1203.5398 [hep-ph].

[34] W. Ochs, Nucl. Phys. Proc. Suppl. 174 (2007) 146 [hep-ph/0609207]. 